\title{
Numerical Simulation of Interior Fluid For Shock Wave-opening Launching
}

\author{
Aolin Zhang ${ }^{1}$, , Yi Jiang ${ }^{1,}$ Chengde Wang ${ }^{1}$ and Yongyue $\mathrm{Li}^{1}$ \\ ${ }^{1}$ Department of Aerospace Engineering, Beijing Institute of Technology, Beijing, 100081, China \\ aolymdream@163.com
}

Keywords: shock wave-opening, gas jet, numerical simulation

\begin{abstract}
We report on a simulation of interior flow field in launch box with shock wave-opening technology. A three-dimensional model of the launch box has been established to study the process of shock wave forming and transmitting. The simulation of model was calculated with unsteady standard $k-\varepsilon$ model. The whole process of numerical simulation includes rocket engine ignition, back lid opening, and the shock wave reflection. Through comparison of contours of pressure and species mass, it is found that the shock wave forming of air not the gas breaks the back lid. Pressure transmission curve obtained in simulation can be a reference for the range of practical friable lid opening pressures.
\end{abstract}

\section{Introduction}

With the development of missile launch technology, friable lid technology is becoming a main opening lid method of missile's launch boxes. Gas or shock wave generated by jet flow is used in the technology to break the friable lid into pieces of predetermined size, which will then fall down freely. As a kind of friable lid technology, shock wave-opening technology can avoid ablation and high temperature and high pressure effects on missile and launch box. Therefore, shock wave-opening is more reliable than gas open, is worthwhile to study shock wave-opening technology.

The traditional idea that gas flows' straight effect or missile head impact cause opening of launch box's front lid was widely accepted in china[1-4]. However, Fu's [5] experiments and simulations proved the front lid is crushed by shock wave. Actually, their research didn't focus on the back lid. In 2012, Dang Haiyan et al.[6]researched and found propagation characteristics of shock waves in launch box, especially the effect for opening-pressure of back lid to the front lid. They set the opening time, then record the average pressure of back lid in their researches. On the contrary, the opening pressure is set, then we record the time in the paper, which is more in the line with actual situation.

In our research, an unsteady simulation was established to calculate the variation of flow field in the period during the moment of ignition to the moment of front lid broken. The propagation of shock waves in the launch box and the evidence of breaking lids by shock waves were found in the simulations.

\section{Methodology}

To calculate the flow characteristics of gas, the paper uses the Finite Volume Method to solve the basic equations of turbulence and the equations of the standard $k-\varepsilon$ model.

Continuum equation:

$$
\frac{\partial \rho}{\partial t}+\frac{\partial}{\partial x_{i}}\left(\rho u_{i}\right)=0
$$

Momentum equation:

$$
\frac{\partial}{\partial t}\left(\rho u_{i}\right)+\frac{\partial}{\partial x_{j}}\left(\rho u_{i} u_{j}\right)=-\frac{\partial p}{\partial x_{i}}+\frac{\partial}{\partial x_{j}}\left(\mu \frac{\partial u_{i}}{\partial x_{j}}-\rho \overline{u_{i}^{\prime} u_{j}^{\prime}}\right)+S_{i}
$$

Energy equation: 


$$
\frac{\partial(\rho T)}{\partial t}+\frac{\partial\left(\rho T u_{j}\right)}{\partial x_{j}}=\frac{\partial}{\partial x_{j}}\left(\frac{k}{c_{p}} \frac{\partial T}{\partial x_{j}}-\rho \overline{u_{j}^{\prime} T}\right)+S
$$

The standard $k-\varepsilon$ model is the classic two-equation turbulence model. In the model, the turbulent viscosity $\mu_{t}$ is a function of the turbulent kinetic energy $k$ and the turbulent dissipation rate $\varepsilon$.

The transport equation of $k$ :

$$
\frac{\partial(\rho \varepsilon)}{\partial t}+\frac{\partial\left(\rho \varepsilon u_{i}\right)}{\partial x_{i}}=\frac{\partial}{\partial x_{j}}\left[\left(\mu+\frac{\mu_{t}}{\sigma_{\varepsilon}}\right) \frac{\partial \varepsilon}{\partial x_{j}}\right]+C_{1 \varepsilon} \frac{\varepsilon}{k}\left(G_{k}+C_{3 \varepsilon} G_{b}\right)-C_{2 \varepsilon} \rho \frac{\varepsilon^{2}}{k}+S_{\varepsilon}
$$

The transport equation of $\varepsilon$ :

$$
\frac{\partial(\rho \varepsilon)}{\partial t}+\frac{\partial\left(\rho \varepsilon u_{i}\right)}{\partial x_{i}}=\frac{\partial}{\partial x_{j}}\left[\left(\mu+\frac{\mu_{t}}{\sigma_{\varepsilon}}\right) \frac{\partial \varepsilon}{\partial x_{j}}\right]+C_{1 \varepsilon} \frac{\varepsilon}{k}\left(G_{k}+C_{3 \varepsilon} G_{b}\right)-C_{2 \varepsilon} \rho \frac{\varepsilon^{2}}{k}+S_{\varepsilon}
$$

And the Reynolds stress,

$$
\tau_{i j}=-\rho \overline{u_{i}^{\prime} u_{j}^{\prime}}=\mu_{t}\left(\frac{\partial u_{i}}{\partial x_{j}}+\frac{\partial u_{j}}{\partial x_{i}}\right)-\frac{2}{3}\left(\rho k+\mu_{t} \frac{\partial u_{i}}{\partial x_{i}}\right) \delta_{i j}
$$

\section{Calculation Model}

\section{Initial Conditions and Boundary Conditions.}

Pressure-inlet: the inlet of the engine jet is set to be pressure-inlet. Values of the total temperature and the total pressure are properties of the engine in steady state

Pressure-outlet: the exterior of the whole computational domain is set to be pressure-outlet.

Wall: the surface of the engine jet, the interior of launch box are set to be the stationary wall. The standard wall function is used to deal with wall-bounded turbulent flows.

In the calculation, a CFD software FLUENT has been used and the standard $k-\varepsilon$ model with the standard wall function is employed. Meanwhile, the coupled scheme and the ideal-gas are used. The property of the total pressure of the jet inlet is $2 \mathrm{MPa}$, and that of the total temperature is $3500 \mathrm{~K}$. The property of the environmental pressure is $101325 \mathrm{~Pa}$, and that of the environmental temperature is 300K.The grid number of the model is 270 thousands.

\section{Results and Discussion}

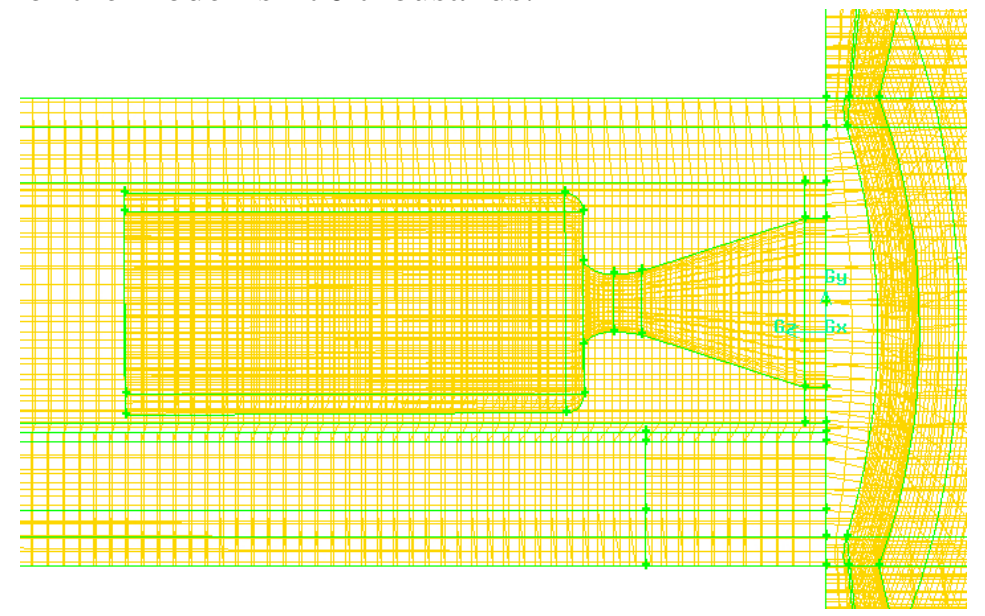

Figure 1. The grid on back lid and rocket engine

A hypothesis was put forward that 2ms (milliseconds) delay replaces the process of back lid opening, in the simulations. In other words, when the average pressure of back lid rising up to the default value, the boundary condition of the lid is transformed from wall condition into interior condition after $2 \mathrm{~ms}$. The opening average pressure value of back lid was designed as $0.17 \mathrm{MPa}$ in a simulation. 


\section{Process of shock wave generation and propagation}

According to the simulation results, at the moment of $0.94 \mathrm{~ms}$, (Defining the moment of engine ignition as the beginning of time), the average pressure of back lid come to the default opening value. After $2 \mathrm{~ms}$, the lid is considered to be crushed fully, and the temperature contour of the launch box at the moment is presented in Figure 2:

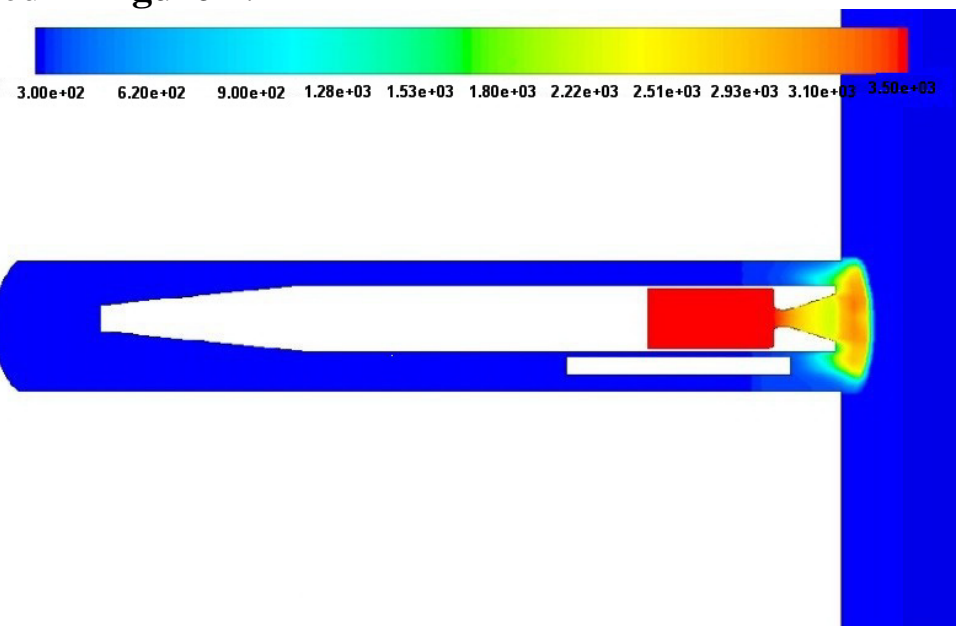

Figure 2. Temperature contour of the launch box at $0.94 \mathrm{~ms}$

A certain amount of gas with high temperature and high pressure has been gathered at the bottom of launch box. It's time to change the boundary condition. With the simulation continuing, the propagation of shock waves in the box can be clearly observed.

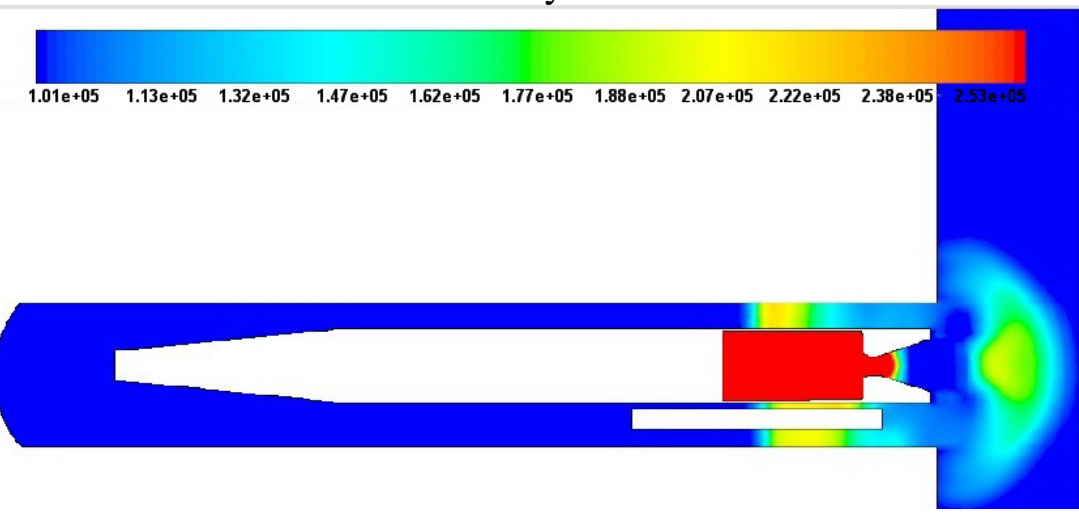

a) .Pressure contour at $4.2 \mathrm{~ms}$

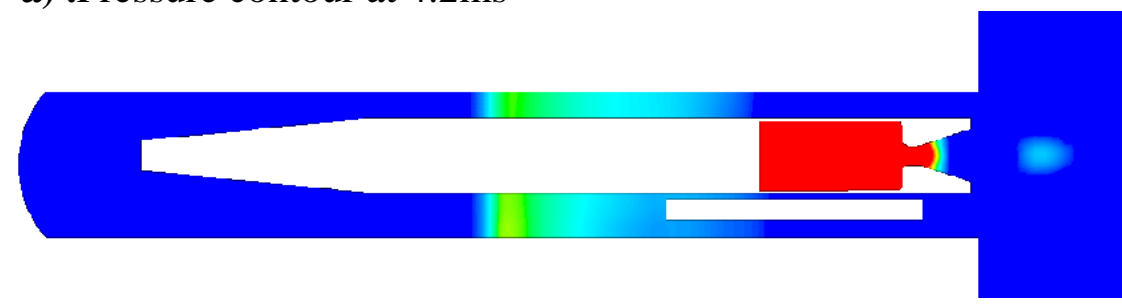

b) . Pressure contour at $9.5 \mathrm{~ms}$

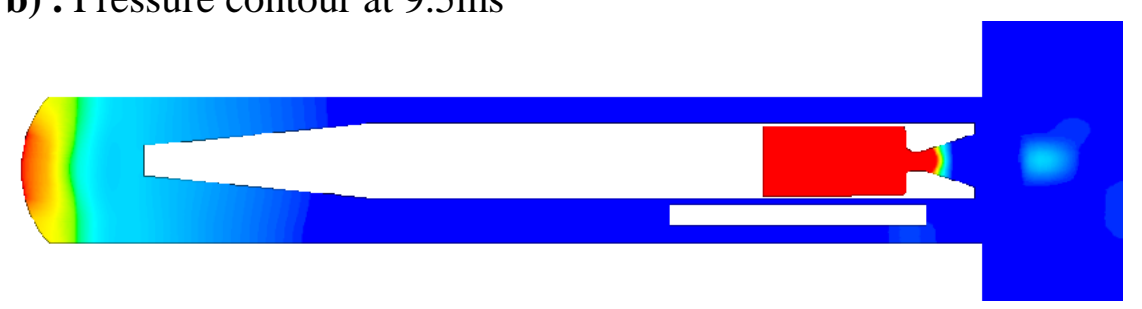

c) . Pressure contour at $18.7 \mathrm{~ms}$

Figure 3. Pressure contours of shock waves’ propagation

After the rocket engine ignition, gas jetted from nozzle condense air in bottom of the launch box, forming shock waves which impact the back lid, then are reflected. Shock waves move forward along 
the gap between the missile and the launch box, reach the front lid, raise the pressure of the front lid, and crush the lid, then the missile can be launched successfully.

Besides, the velocity, temperature and mass of gas contours are recorded in the process of the propagation. For example, there are different contours at the same moment as follows:

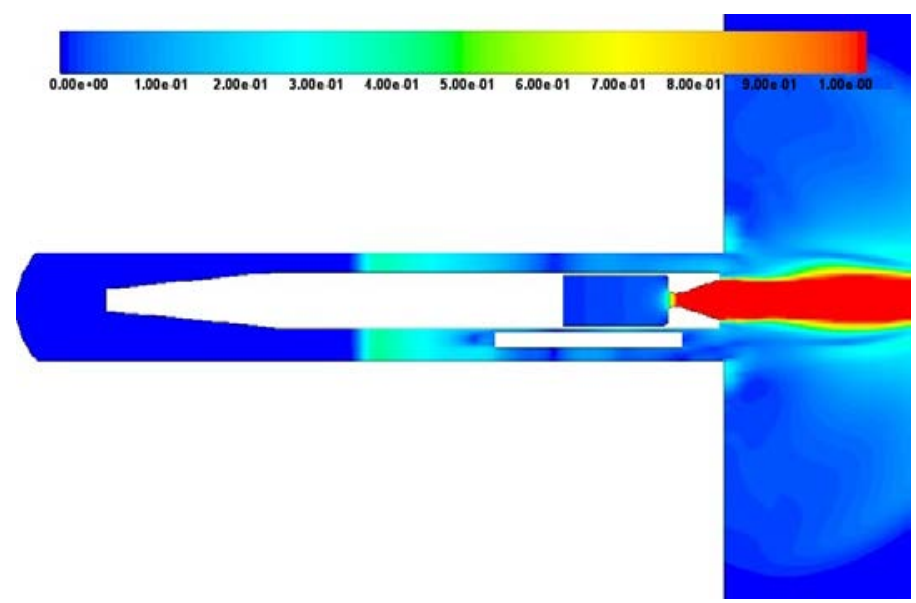

Figure 4. Mach contour at $9.56 \mathrm{~ms}$

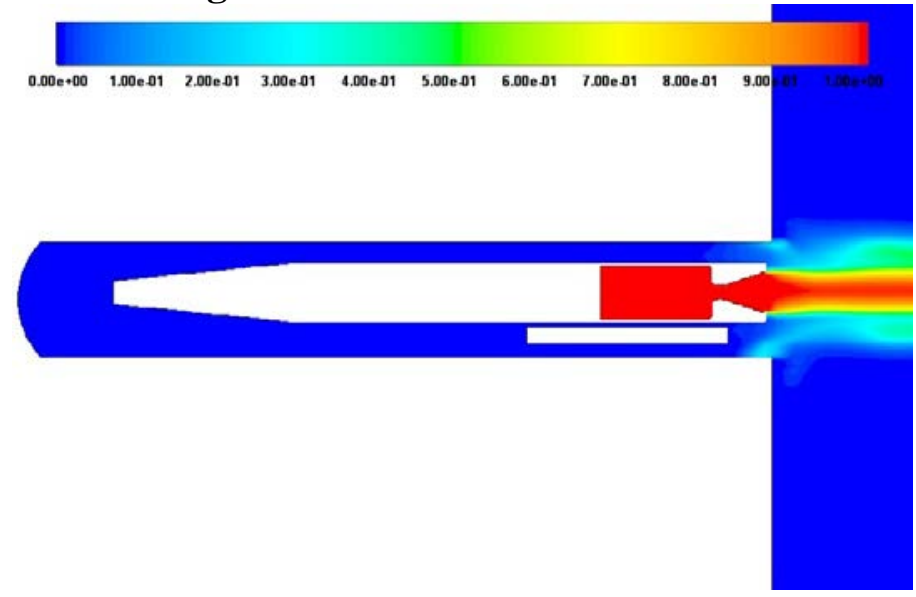

Figure 5. Mass contour of gas at $9.56 \mathrm{~ms}$

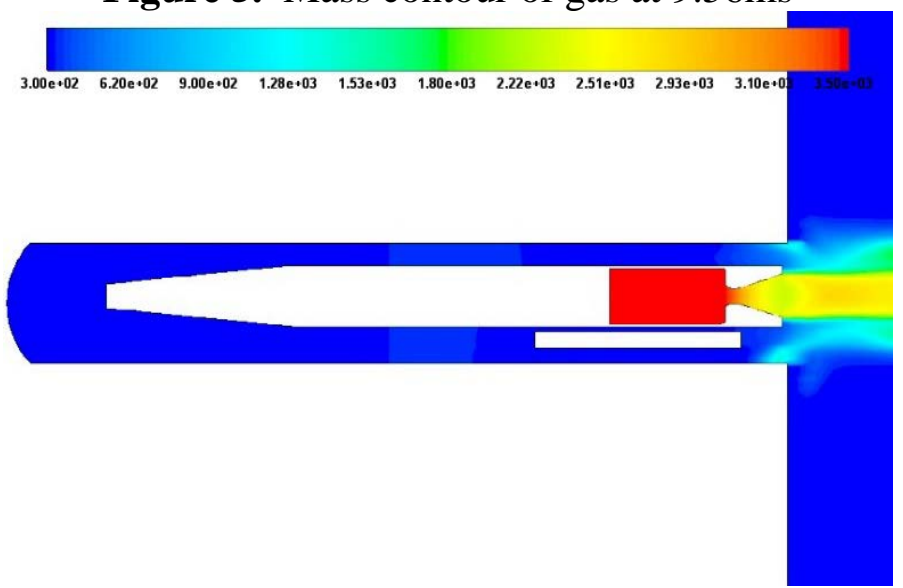

Figure 6. Temperature contour at $9.56 \mathrm{~ms}$

Shown in figures, we can found that:

- Shock wave propagation disturbs to the local gas.( Figure 4.)

- There are hardly any gas in the gap between missile and launch box.( Figure 5.)

- Temperature of launch box and missile surface changes a little.( Figure 6.)

Therefore, it can be concluded that shock wave-opening will not greatly affect missile body, thus effectively protecting the missile. 
Analysis of the pressure of lids

The average pressure of four surfaces that contain back lid, front lid, surfaces from the back lid of $3 \mathrm{~m}$ and $6 \mathrm{~m}$ were monitored and the values were recorded.

Using these datum, curve of pressure could be generated:

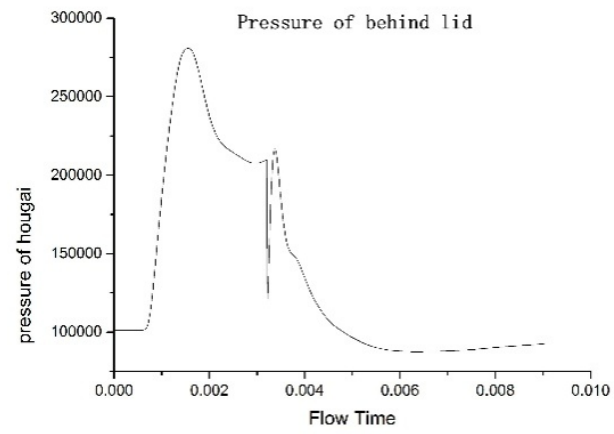

Figure 7. pressure curve of back lid

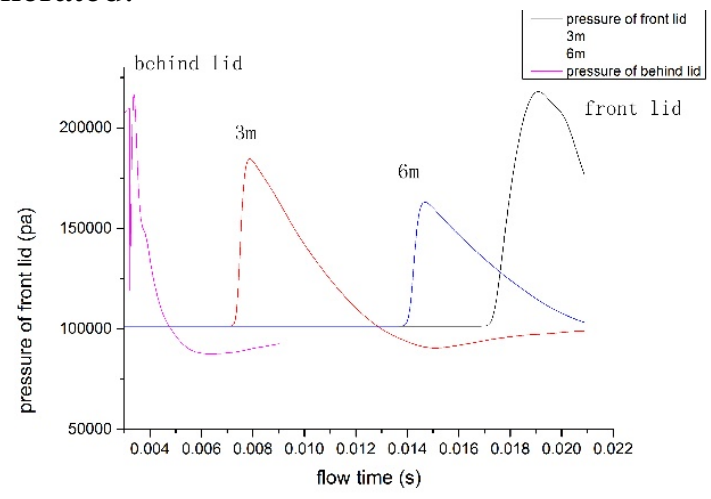

Figure 8. Pressure curves of four surfaces.

According to the Figure 7. , the pressure of the back lid reaches the peak and then decline during the $2 \mathrm{~ms}$-delay, and the peak time is $1.56 \mathrm{~ms}$ with the pressure of $279876 \mathrm{~Pa}$. Changing the boundary condition at the end of 2ms-delay makes the curve instantaneously dropping. Then, the gases are jetted to external basin, with the pressure of the back lid decreasing.

It is shown in Figure 8. that energy of shock waves is consumed in the propagation, with peak pressures significantly decreasing in the surfaces of 3 meters and 6meters. The average velocity of shock wave transmission is $453 \mathrm{~m} / \mathrm{s}$ (meters per second), which can be calculated by the propagation time and the distance between the front and the back lid. The value can be used to design the opening pressures of the front and the back lid.

Evidences of breaking lids by shock waves

As mentioned, Fu's research didn't pay attention to the back lid, there are the evidences of shock wave opening as follows:

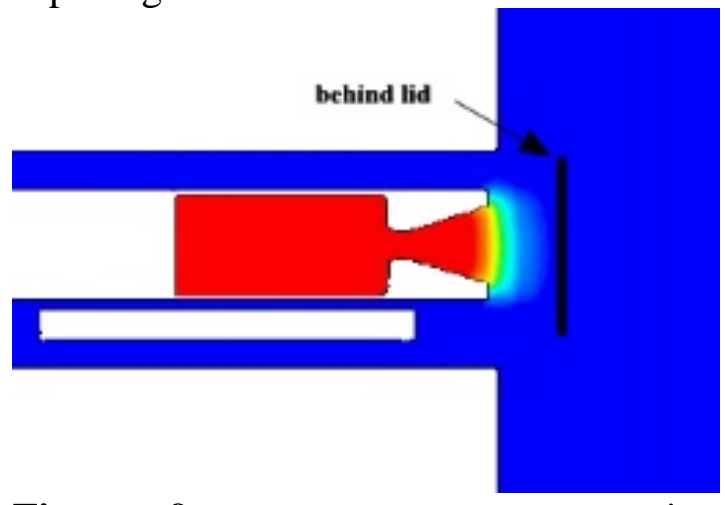

Figure 9. mass contour at opening moment

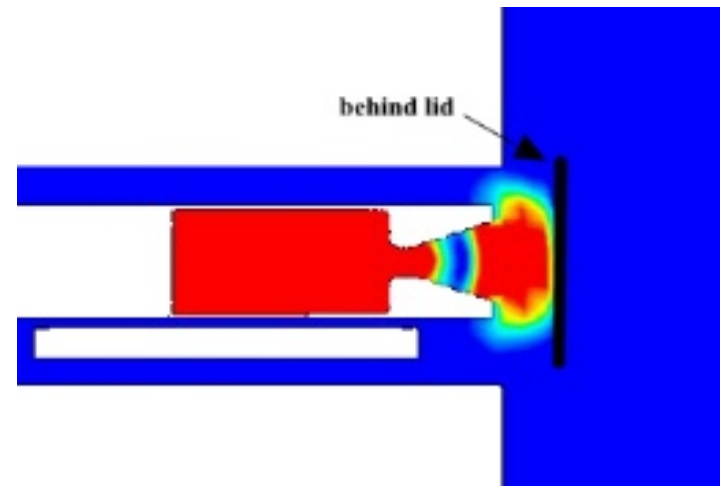

Figure 10 pressure contour at opening moment.

Figure 9. and Figure 10. show that the high pressure representing shock waves reach the back lid, while a little of gases flow out of the nozzle. Thus, the back lid is broken just by shock waves.

\section{Conclusions}

This paper has presented the numerical simulation of shock wave-opening launching, and our findings can be concluded:

The shock wave formation and propagation process in launch box have been obtained;

The simulation analysis shows that the back lid is broken by shock waves rather than the influence of gas; 
The front and the back lid pressure distribution and shock wave propagation velocity can be used as references to design the opening pressures of the front and the back lid;

\section{References}

[1] MIAO Pei-yun, YUAN Zeng-feng(2004) Techniques for the Automatic Cover Opening in Concentric Canister Launcher. Transactions of Beijing Institute of Technology DOI: 10. 15918/j.t bi t1001 -0645. 2004. 04. 0012

[2] JIANG Yi, GENG Feng ,ZHANG Qiang(2008) Numerical Calculation \& Experiments for the Opening Process of Missile Launcher's Cover. Journal of Ballistics

[3] LIU Qi, FU Debin, JIANG Yi(2005). Unsteady Simulation of Shock Wave in Launcher. Journal of Projectiles,Rockets,Missiles and Guidance

[4] JIN Jianfeng XU Gang(2014). Numerical analysis and experimental validation of pressure wave in launcher box. STRUCTURE \& ENVIRONMENT ENGINEERING 41(5)24-27

[5] Fu De-bin, Jiang Yi (2007),Opening process of friable lid of one missile. Journal of Solid Rocket Technology 30(4)275-277

[6] Dang Haiyan, He Weidong. (2012) Research on the Effect for the Opening-pressure of Post-cover to the Pressure of Pre-cover in the Shock Wave Opening .

[7] John D. Anderson. (2010) Computational Fluid Dynamics. chapter 2. China Machine Press

[8] Wang Funju. (2004) Computational Fluid Dynamics Analysis. Tsinghua University Press 